\title{
Linear and nonlinear opponent color coding
}

\author{
JEROEN G. W. RAAIJMAKERS and CHARLES M. M. de WEERT \\ University of Nijmegen, Erasmuslaan 16, Nijmegen, The Netherlands
}

\begin{abstract}
The linearity axiom as proposed by Krantz (1975) is confirmed for red/green equilibria, i.e., lights which appear neither reddish nor greenish (unique yellows, unique blues, and achromatic colors). This experiment also gave some evidence of "veiling," i.e., the masking of a weak hue component by a strong one. In a second experiment, hue magnitude estimates (HME) for the green component were obtained in variable mixtures of unique green and unique yellow stimuli. Contrary to the suggestion raised by the work of Yager and Taylor (1970), we did not find the relation between the HME and the luminance of the green stimulus to be independent of the amount of added yellow. A relation proposed by Indow and Stevens (1966), in which the proportion of the green luminance is taken as the relevant psychophysical unit, gives a better fit to our data. It is shown, however, that alternative interpretations of such data are possible and that this type of experiment cannot be conclusive regarding the issue of the possibility of absolute judgment of opponent hue attributes.
\end{abstract}

Hurvich and Jameson (1957) were the first to propose a quantitative opponent-colors theory based upon the ideas of Hering. Opponent qualities can be quantified by measuring amounts of cancellation energy, i.e., a red stimulus, for example, can be made neither reddish nor greenish by mixing it with an appropriately chosen amount of a green stimulus. In a similar way, yellow can be cancelled by blue.

Krantz $(1974,1975 a$, b) provided a formal theory, based on Hurvich and Jameson's cancellation approach. The set of all possible colored lights contains two subsets, $C_{1}$ and $C_{2}$ (see also Larimer, Krantz, \& Cicerone, 1974). $C_{1}$ is the set of all red/green equilibria, i.e., lights which appear neither reddish nor greenish (unique yellows, unique blues, and achromatic colors). $\mathrm{C}_{2}$ is the set of all yellow/blue equilibria, i.e., lights which appear neither yellowish nor bluish (unique greens, unique reds, and achromatic colors).

The basic assumption made by Krantz is that $C_{1}$ and $C_{2}$ are closed under additive color mixture, denoted by $\Theta$, and under scalar multiplication (insertion of neutral density filters), denoted by *, i.e., if $c$ is in $C_{i}(i=1$ or 2$)$, then

(i) $c \boldsymbol{\oplus} c^{\prime}$ in $C_{i}$ if and only if $c^{\prime}$ is in $C_{i}$,

(ii) if $\mathrm{t} \in \operatorname{Re}^{+}$, then $\mathrm{t} * \mathrm{c}$ is in $\mathrm{C}_{\mathbf{i}}$.

If these axioms and a number of other, less important ones are fulfilled, then any given light can be represented by tristimulus values $\left(\Phi_{1}, \Phi_{2}, \Phi_{3}\right)$ such

We would like to acknowledge the extensive suggestions and comments of David Krantz on the first draft of this paper. Requests for reprints should be sent to: Vakgroep Mathematische Psychologie. Psychologisch Laboratorium, Erasmuslaan 16, Nijmegen, The Netherlands. that a and $b$ are metameric if and only if $\Phi_{i}(a)=\Phi_{i}(b)$ $(i=1,2$, and 3$)$ and

$$
\begin{aligned}
& \text { (i) } \Phi_{i}(a \oplus b)=\Phi_{i}(a)+\Phi_{i}(b), \\
& \text { (ii) } \Phi_{i}(t * a)=t \cdot \Phi_{i}(a) \\
& \text { (iii) } \Phi_{i}(a)=0 \text { if and only if } a \text { in } C_{i} \quad(i=1,2 \text { or } 2) .
\end{aligned}
$$

The functions $\Phi_{1}$ and $\Phi_{2}$ represent the linear (or cancellation) form of the red/green and yellow/blue opponent color codes. They coincide with the chromatic-response functions of Jameson and Hurvich (1955).

The first experiment to be reported concerns these linear opponent color codes and is meant as a test of the linearity axiom, as described above, for $C_{1}$. In this experiment, we determined the amount of energy of a red stimulus necessary to cancel the green in a mixture of a unique green and a unique yellow. Let $a$, $b$, and $c$ be, respectively, the green, the yellow, and the red stimulus. If $a \oplus(t * c)$ is in $C_{1}$, i.e., if $t$ is the cancellation value of a with $\mathrm{c}$ as cancellation stimulus, and if $b$ is in $C_{1}$ (b is a unique yellow), then the above-mentioned axioms (i) and (ii) predict for any $\mathrm{u}, \mathrm{v}$ that

$$
(\mathrm{u} * \mathrm{a}) \oplus(\mathrm{ut} * \mathrm{c}) \oplus(\mathrm{v} * \mathrm{~b}) \text { is in } \mathrm{C}_{1} .
$$

The experiment consisted of varying $u$ and $v$ and determining $w=w(u, v)$ such that

$$
(u * a) \oplus(w * a) \oplus(v * b) \text { is in } C_{1} \text {. }
$$

The prediction is that $w(u, v)=t \cdot u$, where $t$ is a constant; $v$ should have no effect on $w$.

These linear opponent color codes do not necessarily bear direct relations to perceived color 
quantities, for the amount of energy of a green stimulus necessary to cancel a red stimulus does not directly reflect a measure for the amount of perceived redness. just as luminance values do not straightforwardly determine perceived brightness. A major problem in opponent-color theory at this moment is to determine the relation between the linear opponent color codes $\left(\Phi_{1}, \Phi_{2}, \Phi_{3}\right)$ and the codes after (probably nonlinear) transformations which occur later in the visual system (these nonlinear opponent color codes are denoted as $\mathrm{f}_{\mathrm{i}}$ ). This relation presumably is a complex one, simple relations of the form $f_{i}=h\left(\Phi_{i}\right)$ being ruled out for several reasons (Krantz, 1975b). Such a simple form could only be valid if two stimuli which have an equal $\Phi_{i}$ value $(i=$ 1 or 2) also give rise to equal amounts of the corresponding perceived color quality. The existence of "veiling" of a weak hue component by a strong one is used by Krantz as an argument against the simple relation described above.

There are only a few studies on the relation between the nonlinear perceived color quantities and the linear measures of opponent color qualities. These studies furthermore gave quite different results. Yager and Taylor (1970) determined magnitude estimates of yellowness and greenness as functions of luminance of unique yellow and unique green colors, respectively. They found power functions with exponents of about .30 for both the yellow and the green. Indow and Stevens (1966) let subjects estimate the amount of yellowness in a yellow-green mixture of constant total luminance. They found that the magnitude estimates were a power function of the proportion of yellow. Similar measurements were made for other colors. However, since their formulas of the form $\mathrm{a}\left[\left(\mathrm{L}_{1}\right) /\left(\mathrm{L}_{1}+\mathrm{L}_{2}\right)\right]^{b}$ can be rewritten, due to the constancy of $L_{1}+L_{2}$, as $c L_{1} b$, one would expect the exponents to be equal to those of Yager and Taylor. Actually, the exponents of Indow and Stevens were about six times larger than those of Yager and Taylor.

The second experiment is related to both the work of Yager and Taylor and that of Indow and Stevens. We wanted to obtain estimates of the amount of greenness as a function of the luminance of a green stimulus, but under admixture of a variable amount of a unique yellow stimulus, without imposing the condition used by Indow and Stevens that the sum of the yellow and green luminances is constant. Measurements were made at each observer's own unique wavelengths. In the experiment of Indow and Stevens, the same stimuli were used for all observers.

\section{METHODS}

\section{Apparatus}

In Figure 1, a diagram of the equipment is given. Three Carl-Leiss prismmonochromators provided the monochromatic lights. Xenon arc lamps were used as light sources. $M_{1}$ and $M_{2}$ produced green and yellow stimuli. respectively. Intensity of these

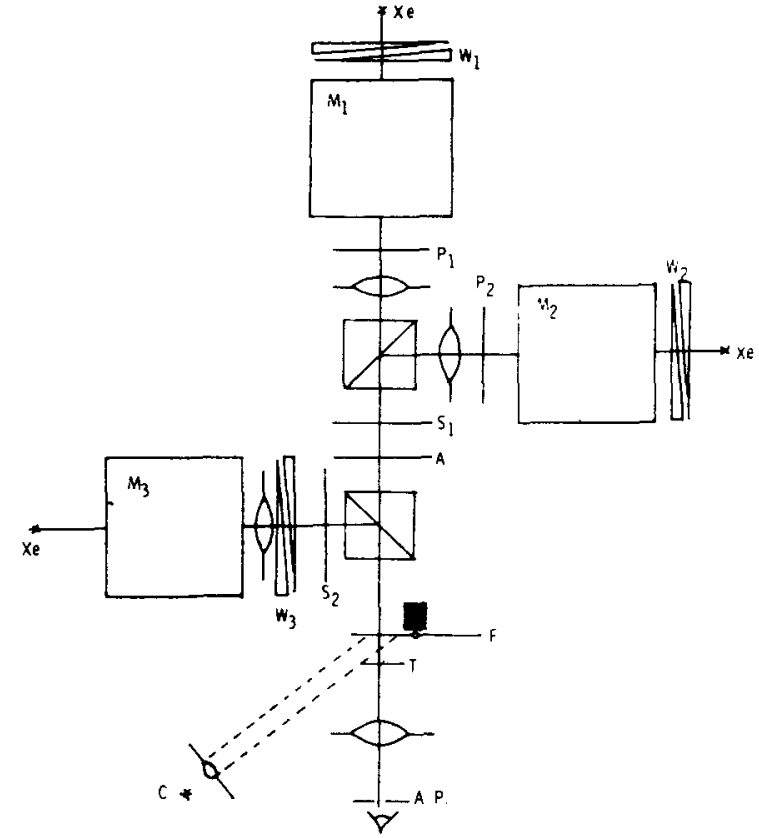

Figure 1. Diagram of the optical equipment: $M_{1}, M_{2}$, and $M_{3}$, prismmonochromators, fed by $X e$ light sources $(150 \mathrm{~W}) ; P_{1}$ and $P_{2}$, orthogonal Polaroids; $A$, analyzer, revolving Polaroid; $S_{1}$ and $S_{2}$, electromagnetically driven shutters; $W_{1}, W_{2}$, and $W_{3}$, compensated circular neutral density wedges; $F$, fickervane; $C$, calbration light sources; T, test target, subtending $2.8^{\circ}$; A.P., 2-mm ctrcubr artificial pupil.

beams was controlled by varying circular neutral density wedges. In Experiment II, a system of orthogonally directed Polaroids $\left(P_{1}\right.$ and $P_{2}$ ) and an analyzer, $A$, were used to control the relative amounts of the yellow and green stimuli. The test target was seen in Maxwellian view and subtended $2.8^{\circ} . M_{3}$ provided the cancellation stimulus in Experiment $I$. $S_{1}$ and $S_{2}$ were electromagnetically driven shutters. with circular diameter of $5 \mathrm{~cm}$. A calibration light, $C$, of constant intensity could be projected upon a flicker vane, $F$, in order to equate the luminance of the test stimuli to a standard. The subject's head rested on a chin and forehead rest. As to the calibration of the Polaroid system, some remarks must be made. Because of different reflection losses for differently polarized lights, the output of the system at the observer's eye cannot simply be computed from $L_{1} \sin ^{2} a+L_{2} \cos ^{2} a$, where $a$ is the difference in polarization angle of $P_{2}$ and $A$. We therefore measured the actual output of both $M_{1}$ and $M_{2}$ for different angles of $A$ separately at the subject's eye. The retinal illumination corresponding to the standard calibration light was 71 troland.

\section{Subjects}

Two subjects, W and P, served in Experiment I. Five subjects, W. P. J. S, and R, took part in Experiment II. For subject W, the experiment was repeated after about 3 weeks. Four of the observers had no technical knowledge about color. The other (W) was experienced in color experiments. but he had never made any hue magnitude estimates. All subjects were screened for color vision defects and found to have normal color vision. All observers used their right eyes.

\section{Determination of Unique Colors}

After a dark-adaptation period, the subjects were instructed about the flickerphotometric procedure in order to be able to equate the test stimuli in luminance against the calibration light. After a few trials, even the inexperienced subjects had no trouble with it. $M_{1}$ and $M_{2}$ were set at about the common wavelengths for 
Table 1

Wavelength Settings for Unique Yellow and Green

\begin{tabular}{ccc}
\hline Subject & Yellow (nm) & Green (nm) \\
\hline W1 & 580 & 516 \\
W2 & 579 & 510 \\
P & 578 & 502 \\
J & 579 & 520 \\
S & 578 & 515 \\
R & 579 & 520 \\
\hline
\end{tabular}

unique green and unique yellow, and these lights were equated in luminance. Next, the yellow (green) stimulus was presented for $1.5 \mathrm{sec}$ in a 15-sec cycle, each presentation being preceded by a 20 -msec click, at $500 \mathrm{msec}$ before the start. During the 13.5 -sec pauses, only a very faintly illuminated ring around the test stimulus was visible. Subjects were asked to fixate the center of that ring after the click was heard. After a rough determination of the wavelength for the unique color, seven wavelength values around it were chosen by the experimenter. These were presented in a random block design, in which each of the seven wavelength values was presented 10 times. The subjects had to say whether red or green was seen in the case of the yellow stimulus and blue or yellow in the case of the green stimulus. The $50 \%-50 \%$ value was taken as the wavelength for the unique color. In Table 1 , the wavelength values for the unique colors are given for the different subjects.

\section{EXPERIMENT I \\ Test of the Linearity Axiom}

The aim of this experiment was twofold. In the first place, we wanted to test the linearity axiom, and secondly, we wondered whether the veiling proposed by $\mathrm{Krantz}$ could also be found in the determination of the amountsof cancellation energy, when the test stimulus is mixed with different amounts of another color component. If veiling of a weak hue component by a strong one occurs, we would expect this to be visible in the cancellation results as well, because in order to be able to cancel a small amount of color, one must be able to detect it: veiling should enlarge the variability in the cancellation amounts. In this experiment, measurements were made of the amounts of a red cancellation stimulus, necessary to neutralize the greenness for a number of levels of the green stimulus at different levels of admixture of unique yellow.

\section{Method}

Procedure. Wavelength values for unique green and yellow stimuli were determined as described above. ${ }^{1}$ Monochromator $M_{3}$ was set at $690 \mathrm{~nm}$, a nonunique red, the intensity of which could be controlled by the observer by turning the knob of a circular neutral density wedge $\left(W_{3}\right)$. In order to be able to make at least an approximate adjustment of the cancellation stimulus within one presentation period of the test stimulus, this period was set at $5 \mathrm{sec}$, followed by a 10 -sec dark period. A click was given $500 \mathrm{msec}$ before the start of the test stimulus. Five to six cycles were necessary in general before the subject was satisfied about the criterion of no redness, no greenness. The different yellow-green combinations were presented at random. Subject $W$ made 6 adjustments at each combination, whereas subject $P$ made 10 adjustments, because of the larger variability in his measurements.
Stimulus levels. At each of six levels of the green stimulus, 7,18 , $35,71,126$, and 142 troland, three levels of yellow were used, 0,71 , and 142 troland. For ease, the 71-troland level is taken as unity.

\section{Results and Discussion}

The results for the two observers are presented in Figure 2. As stated above, under linearity, we predict $\mathrm{w}(\mathrm{u}, \mathrm{v})=\mathrm{t} \cdot \mathrm{u}$, or $\Phi_{1}=\mathrm{t} \cdot$ Lgreen. For the estimation of the slope parameter for both observers, one point, $\mathrm{L}_{\mathrm{gr}}=1.50$ and $\mathrm{L}_{\mathrm{y}}=2.00$, is left out because of the obvious deviancy. It is not clear why it is just this point which deviates so strongly from the expected linear relation. The other points, however, give a very nice fit, thus confirming the linearity axiom for $C_{1}$. The standard deviations in the amounts of the red
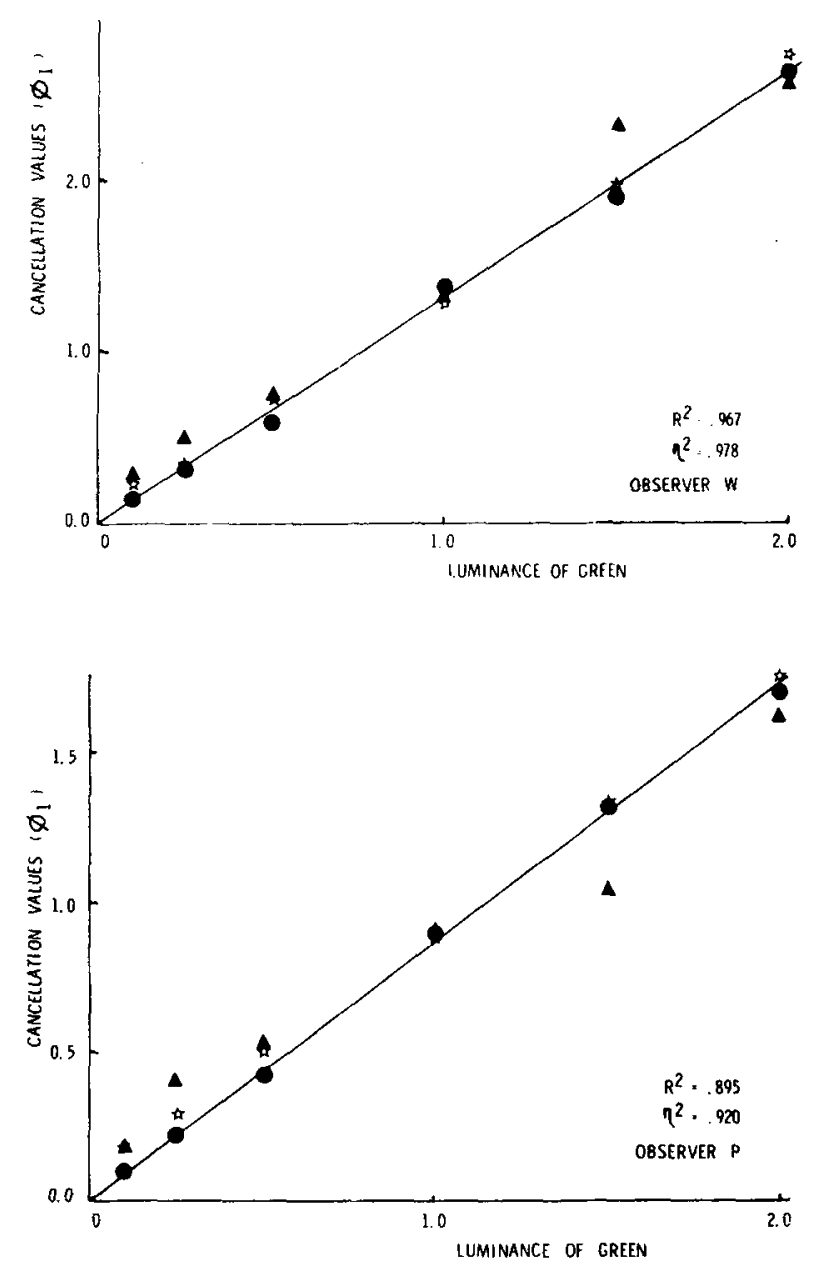

Figure 2. Cancellation amount $\left(\Phi_{1}\right)$ vs. the luminance of the green component in a yellow-green mixture: , no admbed yellow; $*, L$ yellow $=1.00 ; \Delta, L$ yellow $=2.00$. $R^{2}$ gives the proportion of the varlance in the cancellation amounts explained by the formula $\Phi_{1}=t$. Lreen, as specified by opponent-colors theory; $\eta^{2}$ gives the maximum explainable variance, i.e., it takes as a predicted value the mean of values belonging to one particular yellow-green combination. Points are the means of 6 values for observer $W$ and 10 for observer $P$. 
cancellation stimulus increase with increasing luminance of the green stimulus, at a fixed level of yellow admixture, and with increasing amounts of yellow admixture at a fixed level of the green luminance, see Table 2. This can be interpreted as evidence for the occurrence of veiling of small amounts for redness or greenness by whiteness and yellowness, which are formed by the cancellation process itself and by the admixture of a yellow stimulus. Adding yellow does not, however, systematically alter the mean cancellation amounts, such that linearity is disturbed. Using a different design, Larimer et al. (1974) also concluded that linearity holds for red/green equilibria. For the yellow/blue system, matters seem to be more complicated; Larimer, Krantz, and Cicerone (1975) showed that linearity does not strictly hold for yellow/blue equilibria.

\section{EXPERIMENT II \\ Estimation of the Amount of Greenness in Yellow-Green Mixtures}

\section{Method}

Procedure. After the determination of the unique colors, the luminance of the green and yellow lights were equated again at the $0^{\circ}$ and $90^{\circ}$ positions of the analyzer, respectively. Subsequently, the subject was instructed on his task as follows: "Each 15 sec, after a click, a short flash will be given, the color of which varies between yellow and green." The range of colors was then shown to the subject. "Your task is to assign numbers to the amount of greenness present in the flash. To the first flash, you may give any positive number which seems appropriate to you. To the following color, you should give a number proportional to your impression of greenness; if it seems to you that a color has three times the amount of greenness of the first stimulus, you should assign a number to it three times as large as the number given to the first one; if it seems to have one-quarter the amount of greenness of the first stimulus, your number should be one-quarter as large as the first given one." Subjects $S, R$, and $J$ were explicitly asked to ignore the yellowness in the stimulus and also to avoid judgment of the ratio of greenness to yellowness, or of the proportion of greenness. At least three judgments for different levels of greenness were given before the answers were noted by the experimenter. The stimulus presented first was different for the different subjects.

Range of green and yellow luminances. In Table 3, 12 combinations of yellow and green luminances are given. These values were obtained by setting the analyzer at different angles, for two levels of the yellow wedge, $w_{2}$, which differ by a factor of 2.45 . These 12 combinations were presented at random, 10 times each.

Table 2

Standard Deviations in the Cancellation Amounts

\begin{tabular}{cccccccc}
\hline \multirow{2}{*}{$\begin{array}{c}\text { L- } \\
\text { yellow }\end{array}$} & & \multicolumn{7}{c}{ Lgreen } \\
\cline { 3 - 8 } & Subject & .10 & .25 & .50 & 1.00 & 1.50 & 2.00 \\
\hline \multirow{2}{*}{0} & $\mathrm{~W}$ & .01 & .03 & .06 & .08 & .05 & .16 \\
& $\mathrm{P}$ & .01 & .04 & .05 & .12 & .15 & .32 \\
1.0 & $\mathrm{~W}$ & .02 & .10 & .04 & .15 & .15 & .18 \\
& $\mathrm{P}$ & .07 & .09 & .11 & .15 & .23 & .14 \\
2.0 & $\mathrm{~W}$ & .05 & .06 & .11 & .10 & .14 & .42 \\
& $\mathrm{P}$ & .09 & .19 & .22 & .14 & .23 & .35 \\
\hline
\end{tabular}

Table 3

Luminance Values of the Stimuli (in Trolands)

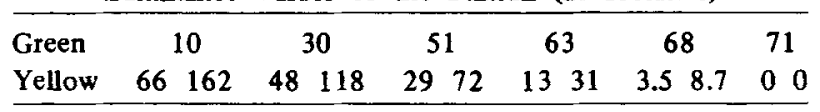

Table 4

Values of the Exponent in Two Power Functions

\begin{tabular}{ccc}
\hline Subject & $(\text { Lgreen })^{\mathbf{b}}$ & $\left(\frac{\text { Lgreen }}{\text { Lgreen + Lyellow }}\right)^{\mathbf{b}}$ \\
\hline W1 & 1,74 & 1,38 \\
W2 & 1,36 & 1,09 \\
P & 1,17 & 1,01 \\
J & 1,12 & 0,89 \\
S & 1,71 & 1,40 \\
R & 1,53 & 1,28 \\
\hline
\end{tabular}

\section{Results and Discussion}

In Figure 3, the results are presented as magnitude estimates against $L_{g r}$ values. Points are the geometrical means of 10 estimates. It is quite clear that admixture of yellow has an important influence on the judgment of the amount of greenness in a mixture of unique green and unique yellow stimuli. This effect is the same for all subjects, whatever type of instruction was used. The amount of greenness is systematically judged to be lower at the higher level of admixed yellow, except of course at the high-luminance side, where the yellow luminance approaches zero. Although, of course, no good fits are obtained when $\log \mathrm{HME}$ are plotted against $\log \mathrm{L}_{\mathrm{gr}}$ values, we still determined the slopes of the best fitting straight lines. These are indicated in Table 4. Even the smallest slopes are much larger than the value of the exponent found by Yager and Taylor. They resemble more those obtained by Indow and Stevens (1966, their Figure 14). They found a value of the exponent of about 1.8. In Figure 4 , we can see that $\log$ HME vs. $\log$ (proportion of green) plots also do not given straight lines, but the deviations from linearity are less than in the $\log \mathrm{L}_{\mathrm{gr}}$ plots. Apart from differences in general form, a more important difference between the two types of plots, is that a single curve can be drawn through the points in Figure 4, and not through the points in Figure 3. The proportion of $\mathrm{L}_{\mathrm{gr}}$ is apparently a better unit for the description of the psychophysical relation than $\mathrm{L}_{\mathrm{gr}}$ alone. This resembles the relation between apparent saturation and colorimetric purity. The huemagnitude-estimation results of Indow and Stevens could possibly be conceived of as functions of the green luminance alone. Our experimental results make it likely that the latter interpretation is incorrect. It may be questioned, however, whether the proportion of the green luminance value in the mixture of yellow and green components is the most relevant unit for the psychophysical description of the hue-magnitude estimates. This argument is related to the well-known insufficiency of the law which 

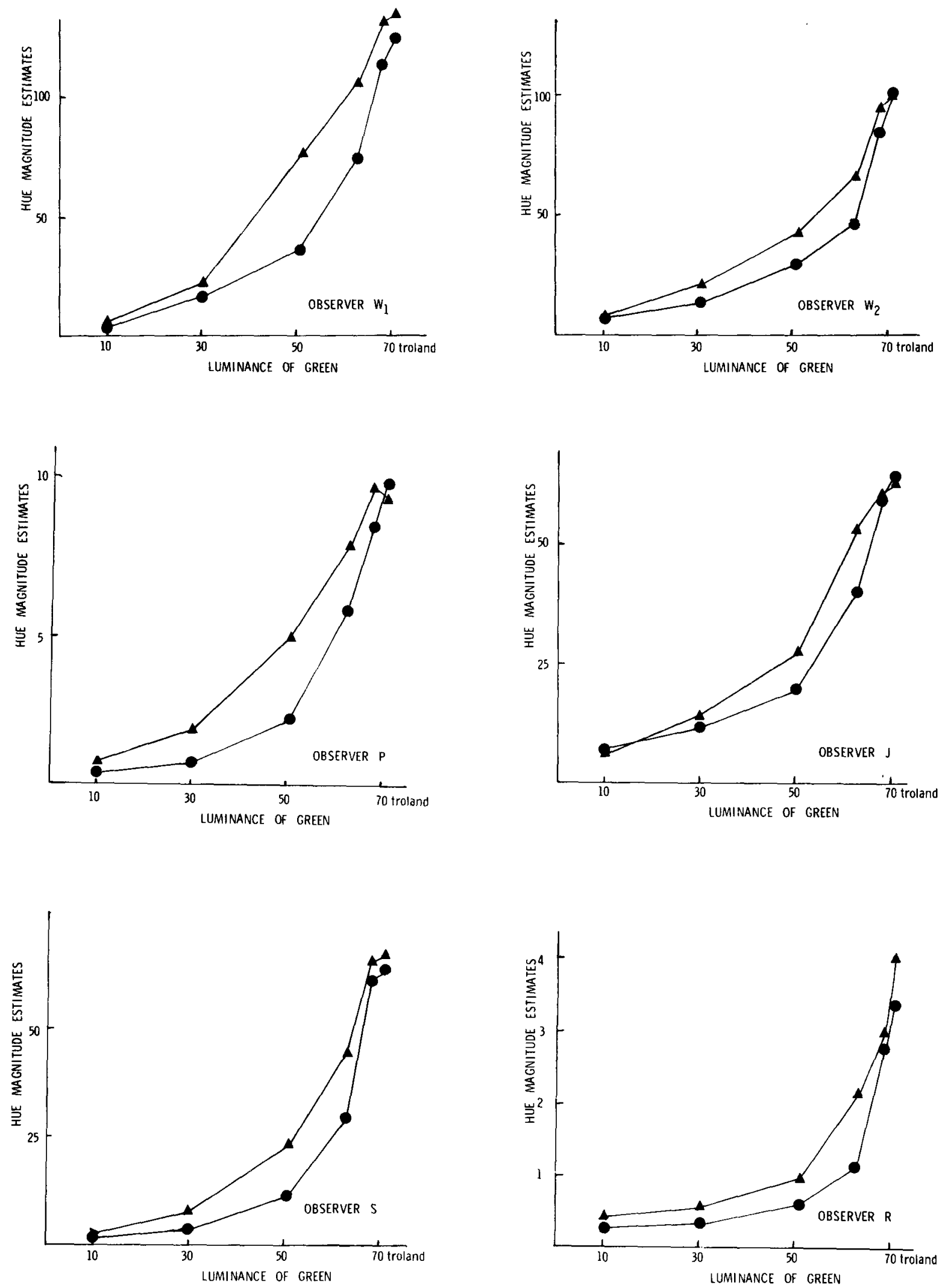

Figure 3. Hue magnitude estimates vs. the luminance of the green component in a yellow-green mixture, for each of six observers. at their own wavelengths for unique green and unique yellow. Points are the geometrical means of 10 estimates: $\bullet$, higher luminance; $\triangle$, lower luminance of the admixed yellow (see Table 3). 

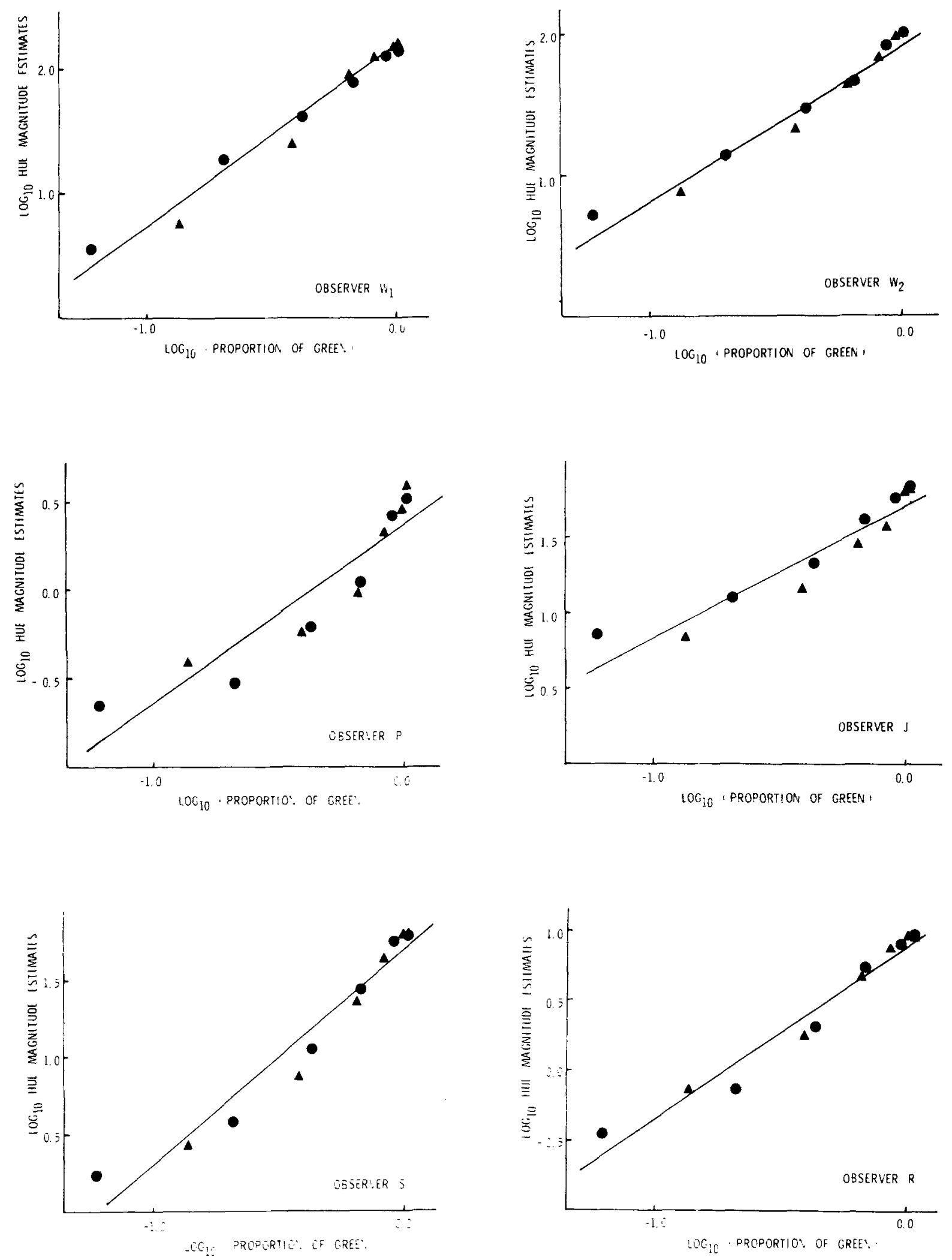

Figure 4. The logarithms of the hue-magnitude estimates vs. the logarithms of the green component in a yellow/green mixture with least squares regression lines for six observers. Points are the geometrical means of 10 estimates: $\bullet$, higher luminance; $\bullet$, lower luminance of the admixed yellow (see Table 30. 
describes the relation between colorimetric purity and apparent saturation for stimuli of different wavelengths. An important problem concerns the interpretation of the finding that relative judgments were given. Did our subjects give relative judgments, due to the fact that our instructions were not effective, or could it be possible that subjects are not able to avoid this kind of relative judgment, or even that the relative strengths of the chromatic and achromatic signals are the only measures available for judgment. In the latter case, the apparent absolute responding obtained by Yager and Taylor could conceivably be explained as relative judgment multiplied by the brightness level-at least one cannot rule out this possibility. This would explain the different course of their functions at lower luminance levels.

In commenting on an earlier draft of this paper, Krantz pointed out some difficulties with this view. First, special status is given to brightness, since one assumes that absolute judgment is possible for brightness but not for opponent hue attributes. Next, the "veiling" phenomenon would become ambiguous. As stated by Krantz: "There are only changes in saturation or in hue, as relative amounts of chromatic attributes and whiteness are varied. The whole question of veiling is predicated on the notion that Hering's opponent qualities can at least be judged ordinally, like brightness, abstracting from the other qualities." Further research should be more conclusive regarding this issue.

One might hope to obtain absolute scales for $f_{i}$ by collecting data in the form of percentage estimates. If one assumes that these are related to the $f_{i}$ values as

$$
H_{i}=\frac{f_{i}}{f_{1}+f_{2}+f_{3}} \quad(i=1 \text { or } 2)
$$

then the following distributive conjoint measurement model would apply:

$$
\frac{1-H_{1}}{H_{1}}=\frac{f_{2}+f_{3}}{f_{1}} .
$$

A similar approach was suggested by Krantz and Tversky (1971, p. 154) for saturation.

\section{REFERENCES}

HURvice, L. M., \& Jameson, D. An opponent-process theory of color vision. Psychological Review, 1957, 64, 384-404.

Indow, T., \& Stevens, S. S. Scaling of saturation and hue. Perception \& Psychophysics, 1966, 1, 253-271.

JAMESON, D., \& HuRvich, L. M. Some quantitative aspects of an opponent-colors theory. I. Chromatic responses and spectral saturation. Journal of the Optical Society of America, 1955, 45, 546-552.

KRANTZ, D, H. Measurement theory and qualitative laws in psychophysics. In D. Krantz et al. (Eds.), Contemporary developments in mathematical psychology (Vol. II). San Francisco: Freeman, 1974. Pp. 160-199.

Krantz. D. H. Color measurement and color theory. I. Representation theorem for Grassmann structures. Journal of Mathematical Psychology. 1975, 12, 283-303.

KRANTz. D. H. Color measurement and color theory. II. Opponent-colors theory. Journal of Mathematical Psychology, $1975,12,304-327$.

KRantz, D. H., \& TVERSKY, A. Conjoint-measurement analysis of composition rules in psychology. Psychological Review, 1971, 78, 151-169.

Larimer, J., Krantz, D. H., \& Cicerone, C. C. Opponentprocess additivity-I: Red/green equilibria. Vision Research, 1974, 14. 1127-1140.

Larimer, J., Krantz, D. H., \& Cicerone, C. C. Opponentprocess additivity-II: Yellow/blue equilibria and nonlinear models. Vision Research, 1975, 15, 723-731.

YAGER, D., \& TAYLOR, E. Experimental measures and theoretical account of hue scaling as a function of luminance. Perception \& Psychophysics, 1970, 7, 360-364.

\section{NOTE}

1. See Table 1 for $W 2$ and $P$.

(Received for publication December 20, 1974; revision received September 4, 1975.) 